\title{
Facile Functionalization of a Metal Carbon Bond by O-Atom Transfer
}

Brian L. Conley ${ }^{\ddagger}$, Somesh K. Ganesh ${ }^{\ddagger}$, Jason Gonzales ${ }^{\dagger}$, William J. Tenn, III $^{\ddagger}$, Kenneth J. H. Young ${ }^{\ddagger}$, Jonas Oxgaard ${ }^{\dagger}$, William

A. Goddard, III ${ }^{\dagger}$ and Roy A. Periana ${ }^{\ddagger} *$

Donald P. and Katherine B. Loker Hydrocarbon Research Institute and Department of Chemistry, University of Southern California, Los Angeles, California 90089, and Materials and Process Simulation Center, Beckman Institute, Division of Chemistry and Chemical Engineering, California Institute of Technology, Pasadena, California 91125.

General Considerations: All air and water sensitive procedures were carried out either in a MBraun inert atmosphere glove box under $\mathrm{N}_{2}$, or using standard Schlenk techniques under argon. Pyridine oxide was purified by sublimation. Labeled reagents $\mathrm{H}_{2}{ }^{18} \mathrm{O}$ (Cambridge Isotopes) and $\mathrm{CH}_{3}{ }^{18} \mathrm{OH}$ (Sigma-Aldrich) were used as purchased. Methyltrioxorhenium was purchased from Strem . GC/MS analysis was performed on a Shimadzu GC-MS QP5000 (ver. 2) equipped with cross-linked methyl silicone gum capillary column (DB5). The retention times of the products were confirmed by comparison to authentic samples. NMR spectra were obtained on a Varian Mercury-400 spectrometer at room temperature. All chemical shifts are reported in units of ppm and referenced to the residual protonated solvent.

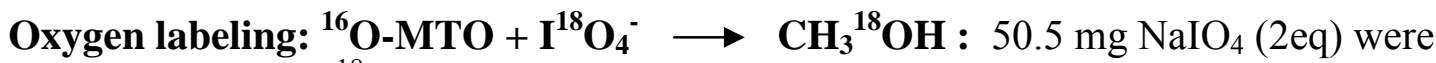
added to $0.5 \mathrm{~mL} \mathrm{H}_{2}{ }^{18} \mathrm{O}$ and allowed to equilibrate for 30 minutes at room temperature with sonication (the exchange of ${ }^{16} \mathrm{O}$ for ${ }^{18} \mathrm{O}$ under such conditions is extremely fast ${ }^{1}$ ). $25.2 \mathrm{mg}$ MTO were added directly to this solution. The reaction quickly turned yellow, then clear within one minute of addition. The reaction mixture was allowed to sit at room temperature for 10 minutes. $1 \mu \mathrm{L}$ was analyzed by $\mathrm{GC} / \mathrm{MS}$ for methanol content. The fragmentation pattern of methanol produced from the reaction was compared to patterns for $\mathrm{CH}_{3}{ }^{18} \mathrm{OH}$ and $\mathrm{CH}_{3}{ }^{16} \mathrm{OH}$. Appearance and relative intensities of peaks in the fragmentation pattern matched that of $\mathrm{CH}_{3}{ }^{18} \mathrm{OH}$. No evidence of $\mathrm{CH}_{3}{ }^{16} \mathrm{OH}$ was present by comparison of the fragmentation pattern to known mixtures of $\mathrm{CH}_{3}{ }^{18} \mathrm{OH}$ and $\mathrm{CH}_{3}{ }^{16} \mathrm{OH}$. The rate of ${ }^{18} \mathrm{O}$ incorporation into MTO was slow relative to the incorporation into $\mathrm{IO}_{4}{ }^{-}$and the production of methanol as measured by GC/MS. 


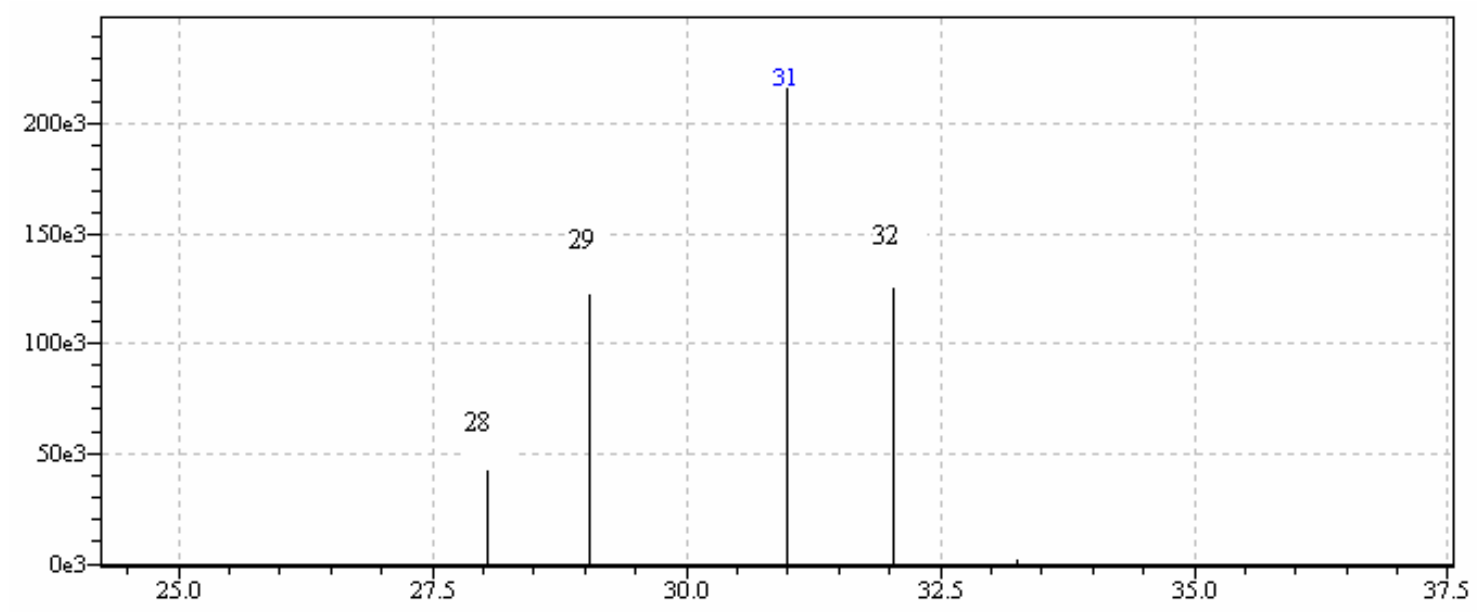

Figure 1: Fragmentation pattern for $\mathrm{CH}_{3}{ }^{16} \mathrm{OH}$ in $\mathrm{H}_{2} \mathrm{O}$ (absolute intensity vs. $\mathrm{m} / \mathrm{z}$ ).

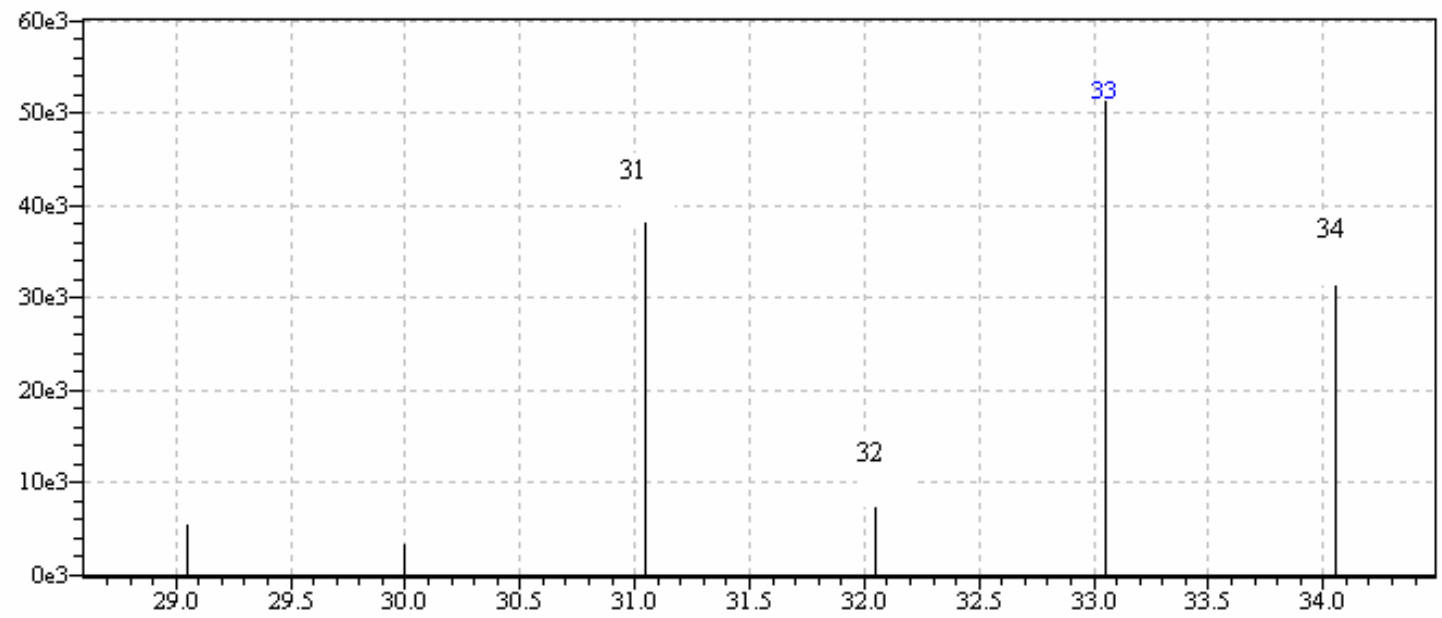

Figure 2: Fragmentation pattern for $\mathrm{CH}_{3}{ }^{18} \mathrm{OH}$ in $\mathrm{H}_{2} \mathrm{O}$ (absolute intensity vs. $\mathrm{m} / \mathrm{z}$ ).

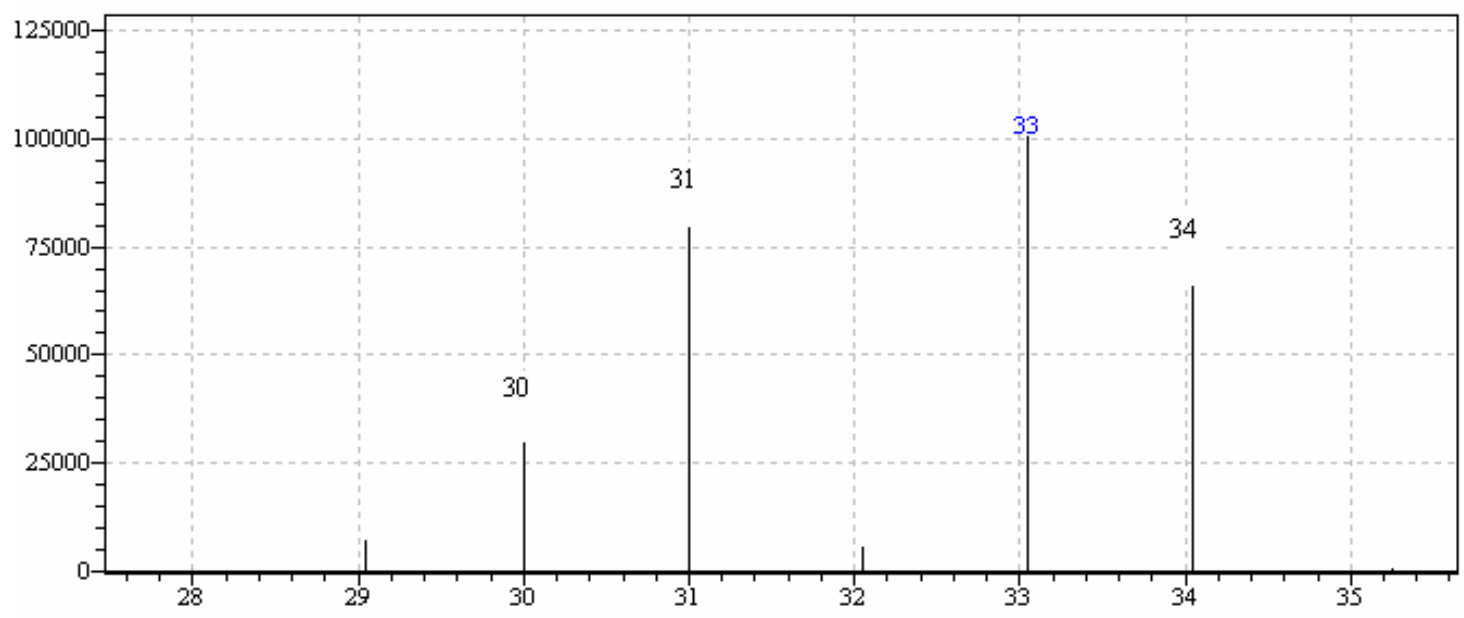

Figure 3: Fragmentation pattern for $\mathrm{CH}_{3}{ }^{18} \mathrm{OH}$ from $\mathrm{MTO}+\mathrm{NaI}^{18} \mathrm{O}_{4}$ reaction in $\mathrm{H}_{2}{ }^{18} \mathrm{O}$ (absolute intensity vs. $\mathrm{m} / \mathrm{z}$ ). 
${ }^{18} \mathrm{O}$-MTO labeling: ${ }^{16} \mathrm{O}$-MTO $+\mathrm{H}_{\mathbf{2}}{ }^{18} \mathrm{O}$ : To verify that the incorporation of ${ }^{18} \mathrm{O}$ into MTO was slow compared to the incorporation into the oxidant, $\mathrm{IO}_{4}{ }^{-}$, we studied this reaction by GC/MS. $28.5 \mathrm{mg}$ MTO was dried thoroughly in vacuo inside a flame dried schlenk tube, sealed with a Chemglass Teflon valve. $1 \mathrm{~g} \mathrm{H}_{2}{ }^{18} \mathrm{O}$ was added using standard schlenk technique. The solution was sonicated for 90 minutes to increase the solubility of MTO in solution and promote the incorporation of ${ }^{18} \mathrm{O}$. The solution was analyzed by GC/MS at this point and the fragmentation pattern compared to the blank $\left({ }^{16} \mathrm{MTO}\right.$ in $\mathrm{H}_{2} \mathrm{O}$ ). The solution was then sonicated for 45 minutes and analyzed again by GC/MS. Though the rate of incorporation was not explicitly measured, as this would require deconvolution due to the natural abundance of Re isotopes $\left({ }^{187} \mathrm{Re}: 62.60 \%,{ }^{185} \mathrm{Re}: 37.4 \%\right)$ it is clear from the fragmentation intensities that incorporation is slow relative to the instantaneous reaction of $\mathrm{H}_{2}{ }^{18} \mathrm{O}$ with $\mathrm{IO}_{4}{ }^{-}$at room temperature. Ignoring the $\mathrm{m} / \mathrm{z}=248$ and 250 peaks as possibly having no incorporation (of course $\mathrm{m} / \mathrm{z}=250$ may have one ${ }^{18} \mathrm{O}$ swapped for a ${ }^{16} \mathrm{O}$ atom), one concludes that the lower limit of incorporation at 135 minutes is $57.9 \%$ based on fragmentation intensities.

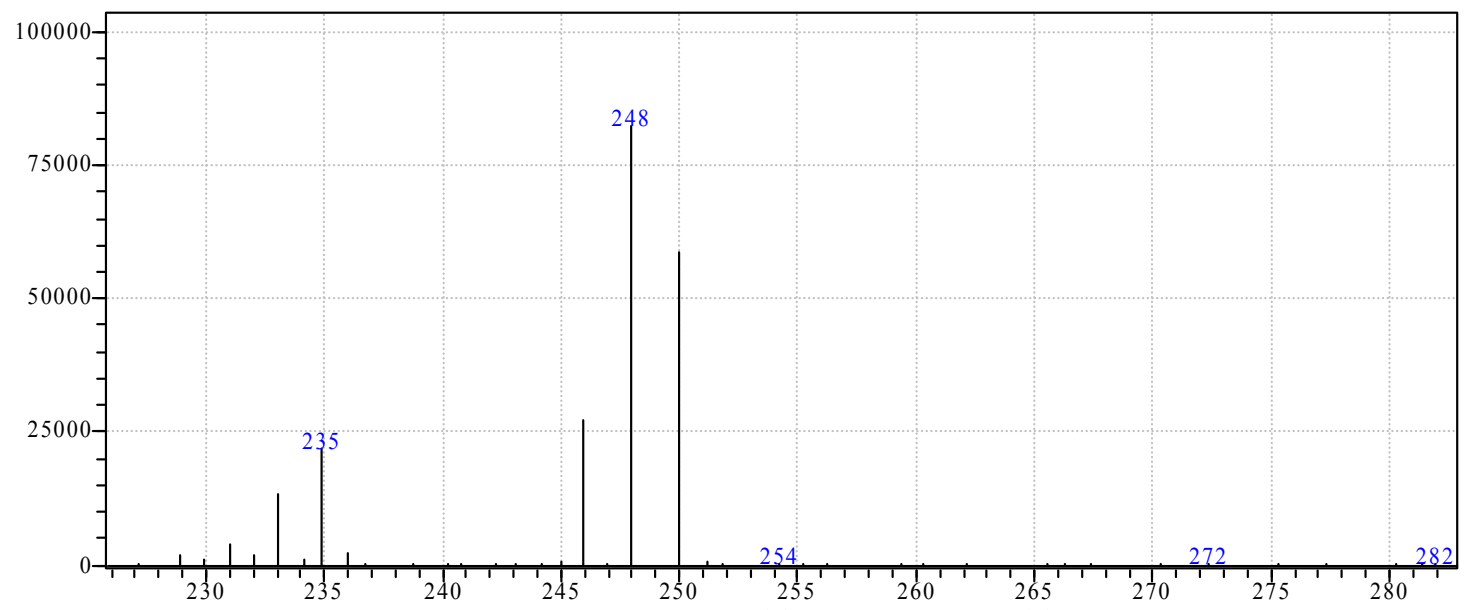

Figure 4: Control. Fragmentation pattern of ${ }^{16} \mathrm{O}-\mathrm{MTO}$ in $\mathrm{H}_{2}{ }^{16} \mathrm{O}$ (absolute intensity vs. $\mathrm{m} / \mathrm{z})$. 


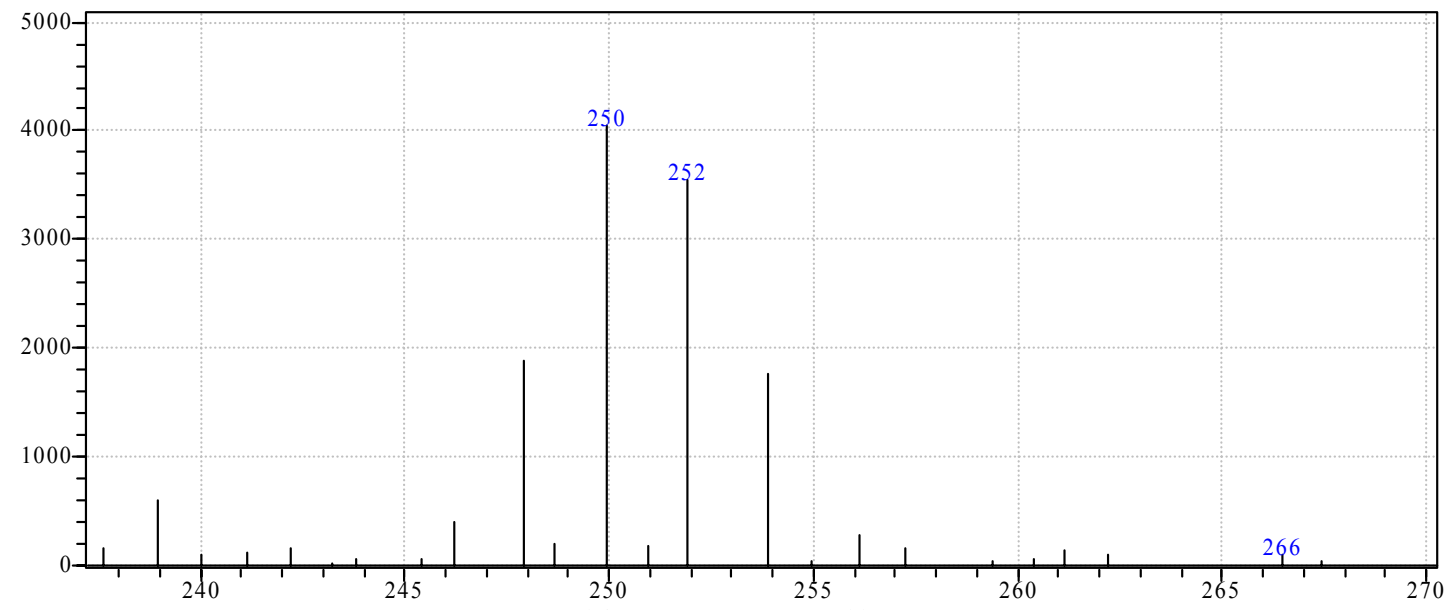

Figure 5: Fragmentation pattern of ${ }^{16} \mathrm{O}-\mathrm{MTO}$ in $\mathrm{H}_{2}{ }^{18} \mathrm{O}$ after 90 minutes(absolute intensity vs. $\mathrm{m} / \mathrm{z}$ ).

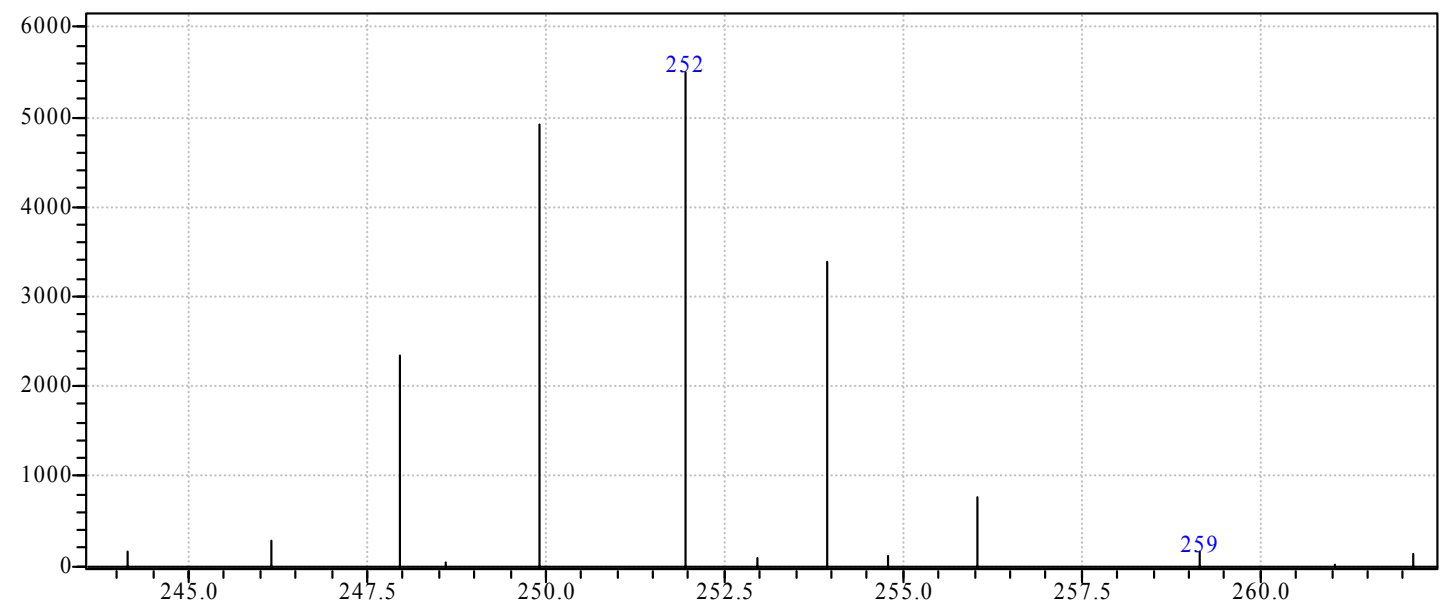

Figure 6: Fragmentation pattern of ${ }^{16} \mathrm{O}-\mathrm{MTO}$ in $\mathrm{H}_{2}{ }^{18} \mathrm{O}$ after 135 minutes (absolute intensity vs. $\mathrm{m} / \mathrm{z}$ ).

$\left(\mathrm{CH}_{3}\right) \mathrm{ReO}_{3}$ (py) + pyO $\longrightarrow \mathbf{C H}_{3} \mathbf{O}-\mathbf{R e O}_{3}$ (py): $12.0 \mathrm{mg}$ MTO were dissolved in $155 \mu \mathrm{L}$ pyridine $\mathrm{d}_{5}$. To this solution was added $0.40 \mathrm{~mL} \mathrm{THF}_{\mathrm{d} 8}, 9.2 \mathrm{mg}$ pyO (2eq), and coaxial capillary containing cyclohexane internal standard $(3.2 \mu \mathrm{L}$ cyclohexane in $50 \mu \mathrm{L}$ $\left.\mathrm{CCl}_{4}\right)$. The ${ }^{1} \mathrm{H}$ NMR was taken at this point $(\mathrm{t}=0)$. The solution was heated at $125^{\circ} \mathrm{C}$ for $1 \mathrm{~h}$. The solution turned orange and precipitated an orange solid on the walls of the JYoung tube. A yield of $40 \%$ was calculated based on conversion of MTO to methoxide, though this does not take into account the precipitated product. No other products were detected by NMR. 


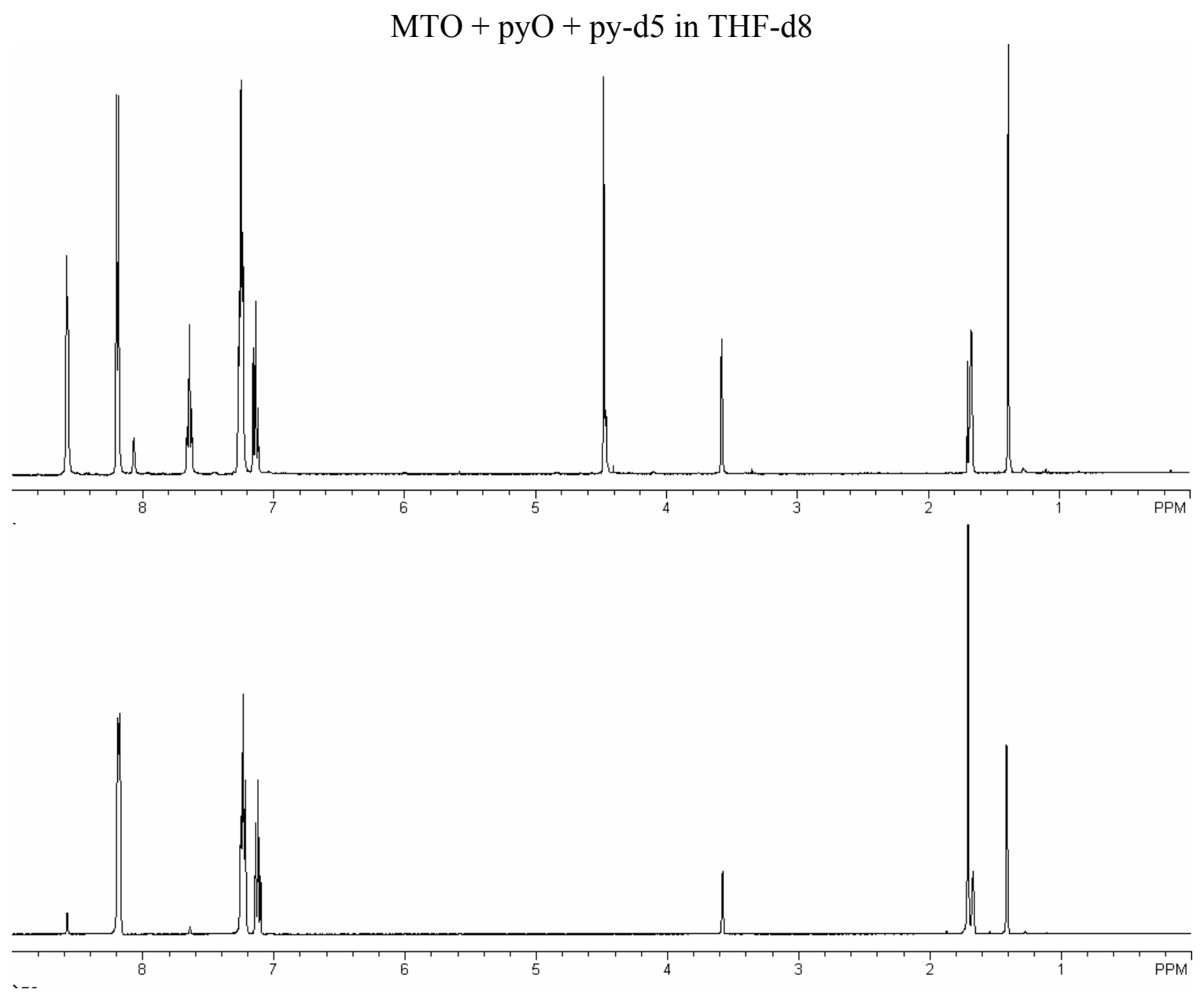

Figure 1: ${ }^{1} \mathrm{H}$ NMR $\left(\mathrm{py}_{\mathrm{d} 5} / \mathrm{THF}_{\mathrm{d} 8}\right)$ of $\mathrm{MTO}+$ pyO reaction at $\mathbf{t}=\mathbf{0}$ (bottom) and $\mathbf{t}=\mathbf{1} \mathbf{h}$ (top): $\delta 3.58$ and $\delta 1.67$ (residual THF resonances); $\delta 8.19\left(\mathrm{~d}, 2 \mathrm{H}, o\right.$-pyO); $\delta 7.25$ (m, overlapping $m$-pyO and pyridine ${ }_{\mathrm{h}}$ ); $\delta 7.12$ (t, $1 \mathrm{H}, p$-pyO); $\delta 8.58\left(\mathrm{~d}, 2 \mathrm{H}\right.$, pyridine $\left._{\mathrm{h}}\right) ; \delta 7.65\left(\mathrm{t}, 1 \mathrm{H}\right.$, pyridine $\left.\mathrm{h}_{5}\right) ; \delta 4.48\left(\mathrm{~s}, \mathrm{CH}_{3} \mathrm{O}-\mathrm{Re}\right) ; \delta 1.70(\mathrm{~s}, 3 \mathrm{H}, \mathrm{MTO}$ methyl); $\delta 1.40$ (cyclohexane I.S.).

$\mathrm{MTO}+\mathrm{YO}+\mathrm{D}_{2} \mathrm{O} \longrightarrow \mathbf{C H}_{3} \mathrm{OH}:$ All reactions were carried out under air in $\mathrm{D}_{2} \mathrm{O}$ in 8 " J-Young NMR tubes. Approximately $16 \mathrm{mg}(0.067 \mathrm{mmol})$ MTO was dissolved in $\mathrm{D}_{2} \mathrm{O}$ with the aid of sonication. 2 equivalents of $Y O$ were added and allowed to react for 1-1.5 hours. The reaction with $\mathrm{OsO}_{4}$ required 2 equivalents of $\mathrm{KOD}$ to produce methanol and thus is reported in Table 1 as $\mathrm{Os}_{4}(\mathrm{OH})_{2}{ }^{2-}$. All appropriate blanks were taken to assign solvent peaks, oxidant peaks, and product (methanol) formation. NMR spectra were obtained on a Varian Mercury 400 spectrometer $\left(400.151 \mathrm{MHz}\right.$ for $\left.{ }^{1} \mathrm{H}\right)$. Chemical shifts are given in ppm relative to residual solvent proton resonances $\left(\mathrm{D}_{2} \mathrm{O}\right.$ at $\left.4.79 \mathrm{ppm}\right)$. Cyclohexane $\left(5 \mu \mathrm{L}\right.$ in $\left.2 \mathrm{~mL} \mathrm{CCl}_{4}\right)$ was used as an external standard. All reactions were carried out under air at room temperature. 


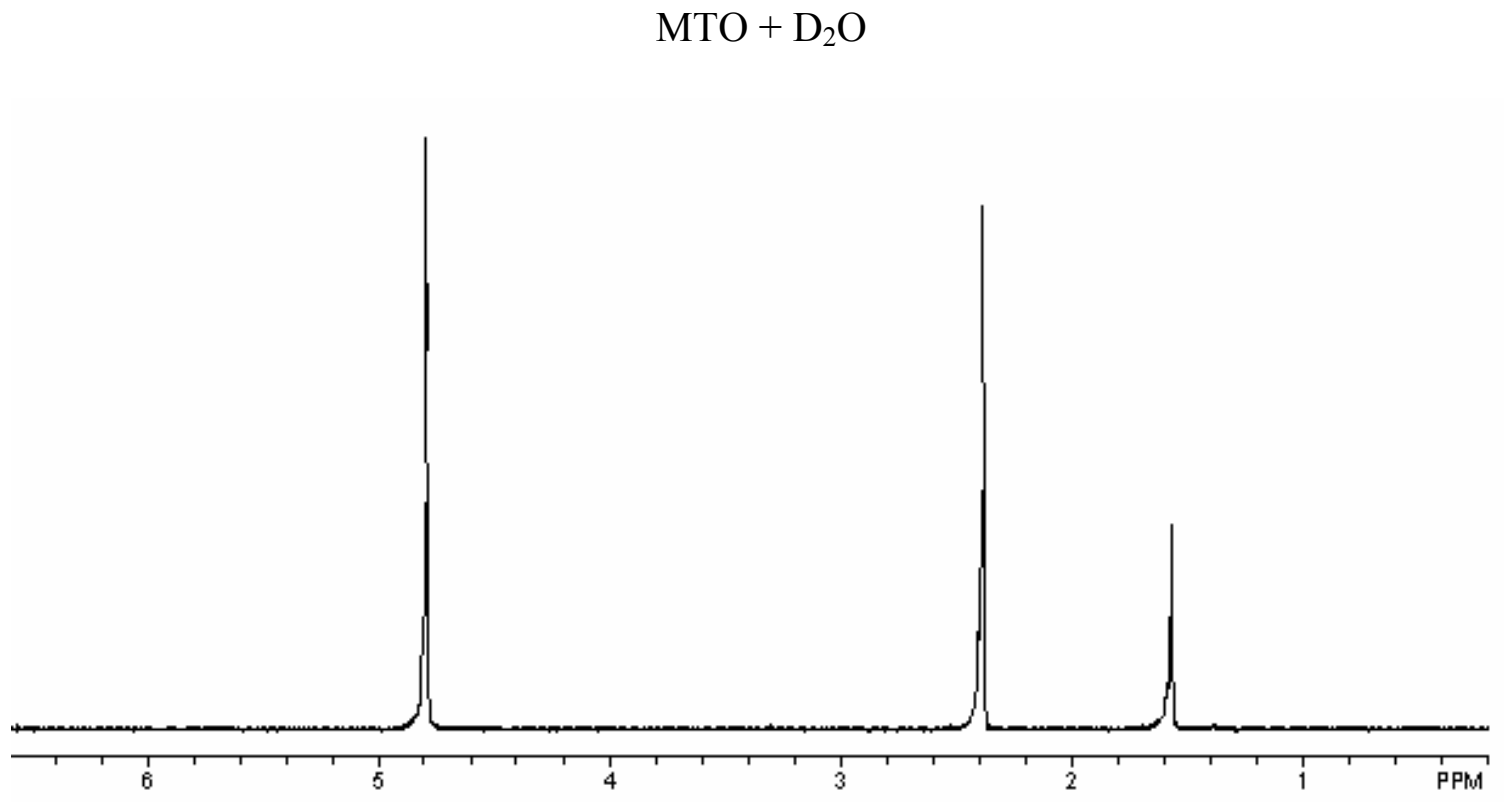

Figure 2: ${ }^{1} \mathrm{H}$ NMR of MTO in $\mathrm{D}_{2} \mathrm{O}$ with cyclohexane internal standard. $\delta 2.38$ (s, 3H, MTO methyl); $\delta 1.57$ (s, cyclohexane I.S.); $\delta 4.79\left(\mathrm{H}_{2} \mathrm{O}\right.$ residual peak).

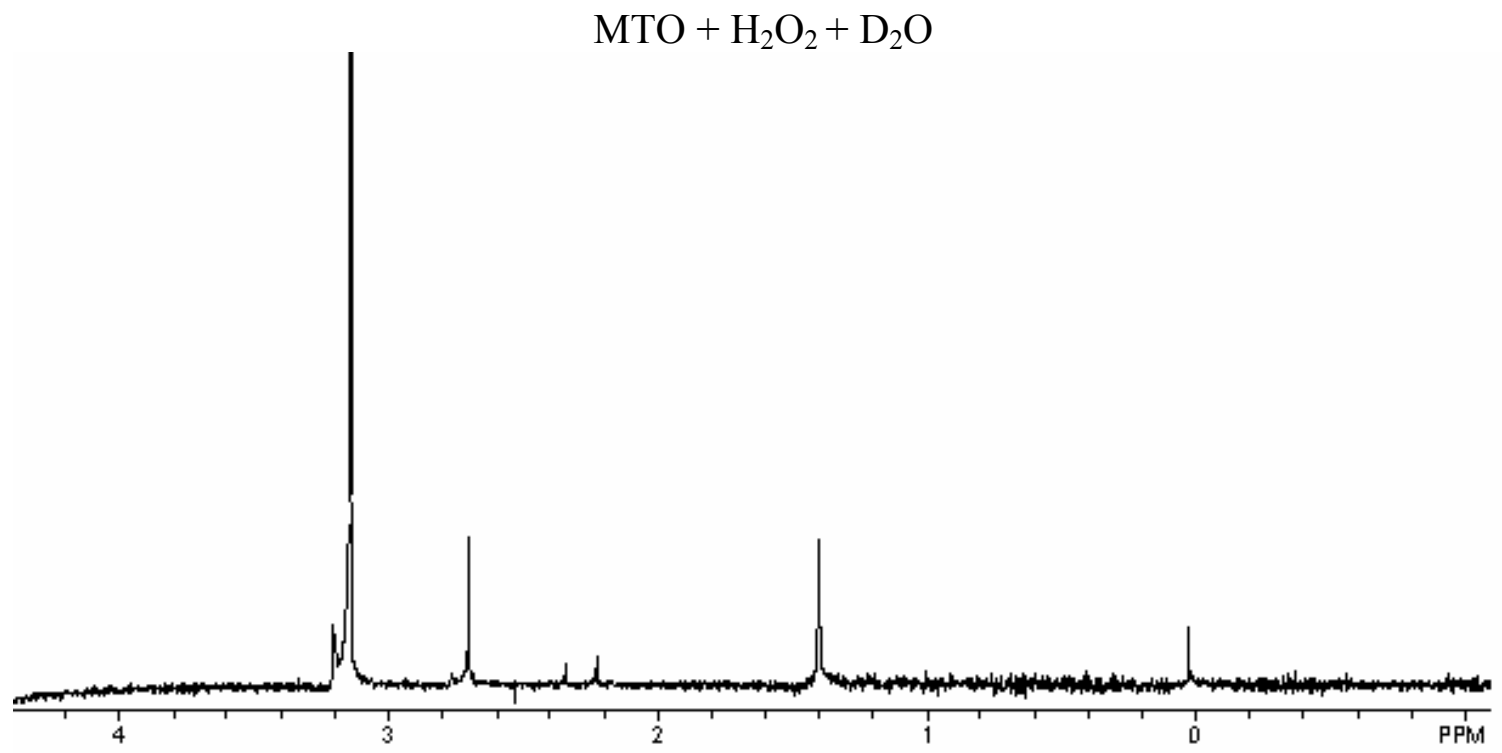

Figure 3: ${ }^{1} \mathrm{H}$ NMR of MTO $+\mathrm{H}_{2} \mathrm{O}_{2}$ in $\mathrm{D}_{2} \mathrm{O}$ with cyclohexane internal standard: $\delta 2.70$ (s, 3H, MTO methyl); $\delta 1.40$ (s, cyclohexane I.S.); 3.14 (s, 3H, methanol). 


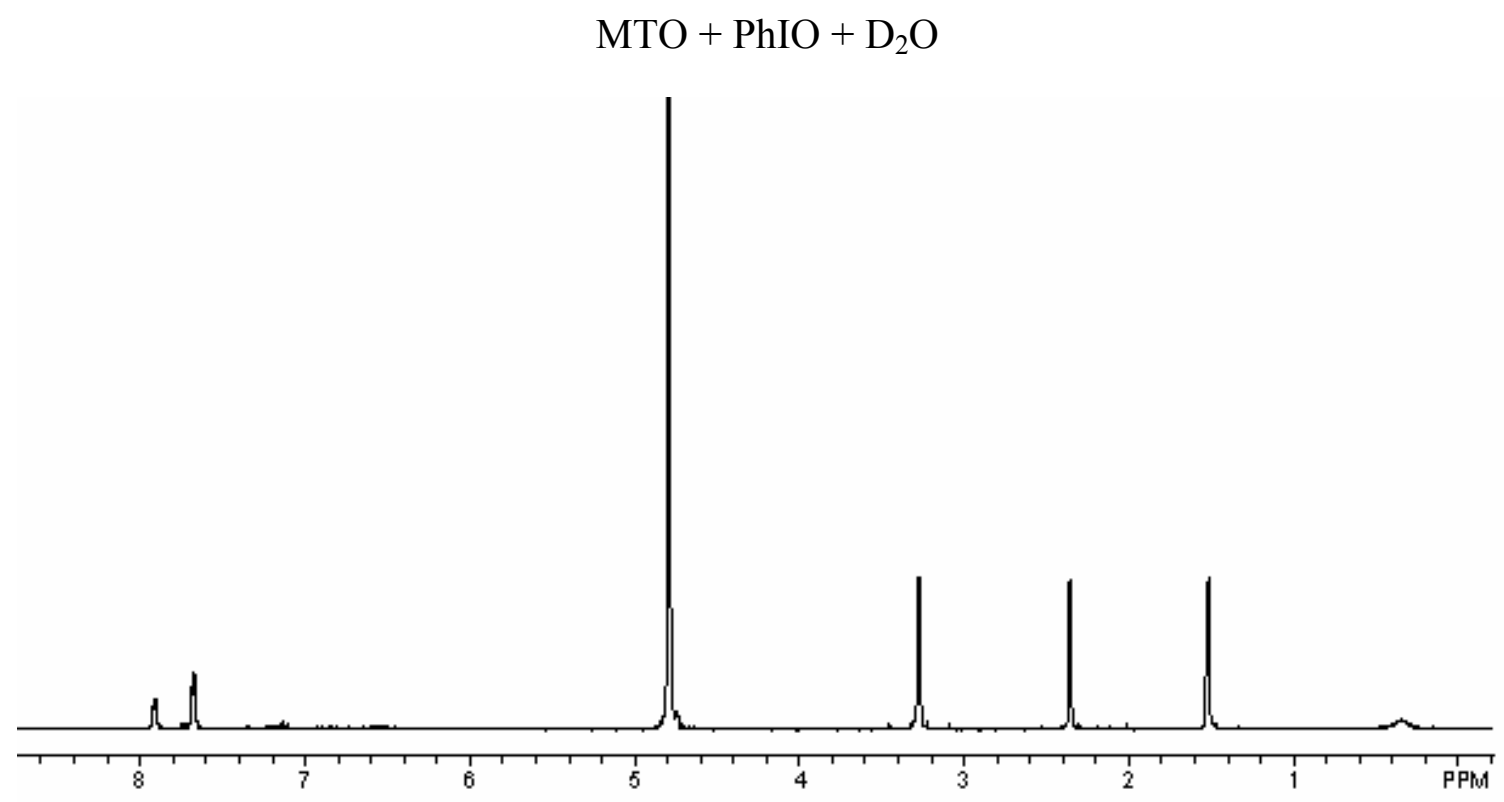

Figure 4: ${ }^{1} \mathrm{H}$ NMR of MTO $+\mathrm{PhIO}$ in $\mathrm{D}_{2} \mathrm{O}$ with cyclohexane internal standard: $\delta 1.52$ (cyclohexane); $\delta$ 2.36 (s, 3H, MTO methyl); $\delta 3.28$ (s, $3 \mathrm{H}$, methanol); $\delta 4.79\left(\mathrm{H}_{2} \mathrm{O}\right.$ residual peak).

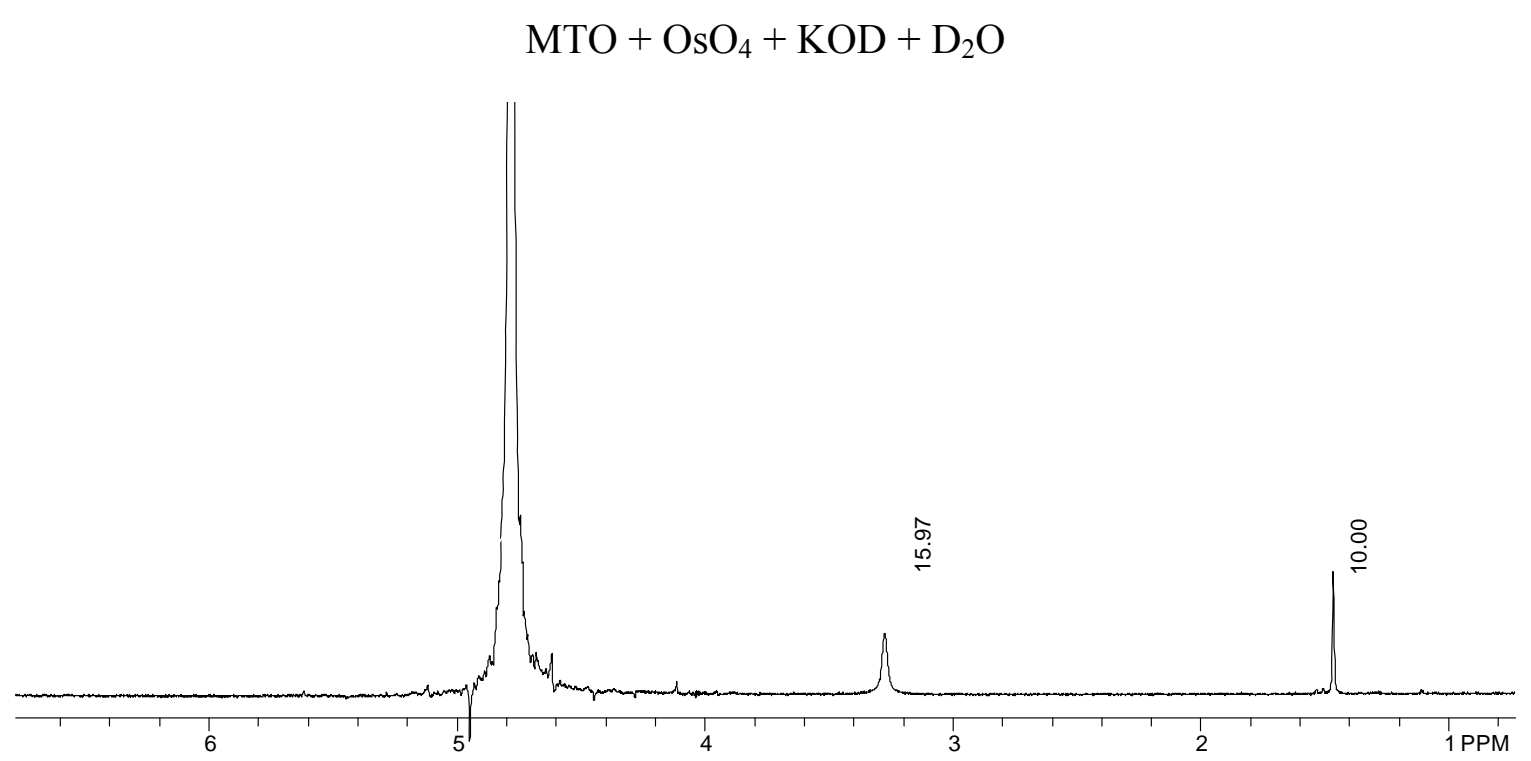

Figure 5: ${ }^{1} \mathrm{H}$ NMR of MTO $+\mathrm{OsO}_{4}$ in $\mathrm{D}_{2} \mathrm{O}$ with added KOD and cyclohexane internal standard: $\delta 1.47$ (cyclohexane); $\delta 3.28$ (s, $3 \mathrm{H}$, methanol); $\delta 4.79\left(\mathrm{H}_{2} \mathrm{O}\right.$ residual peak). 


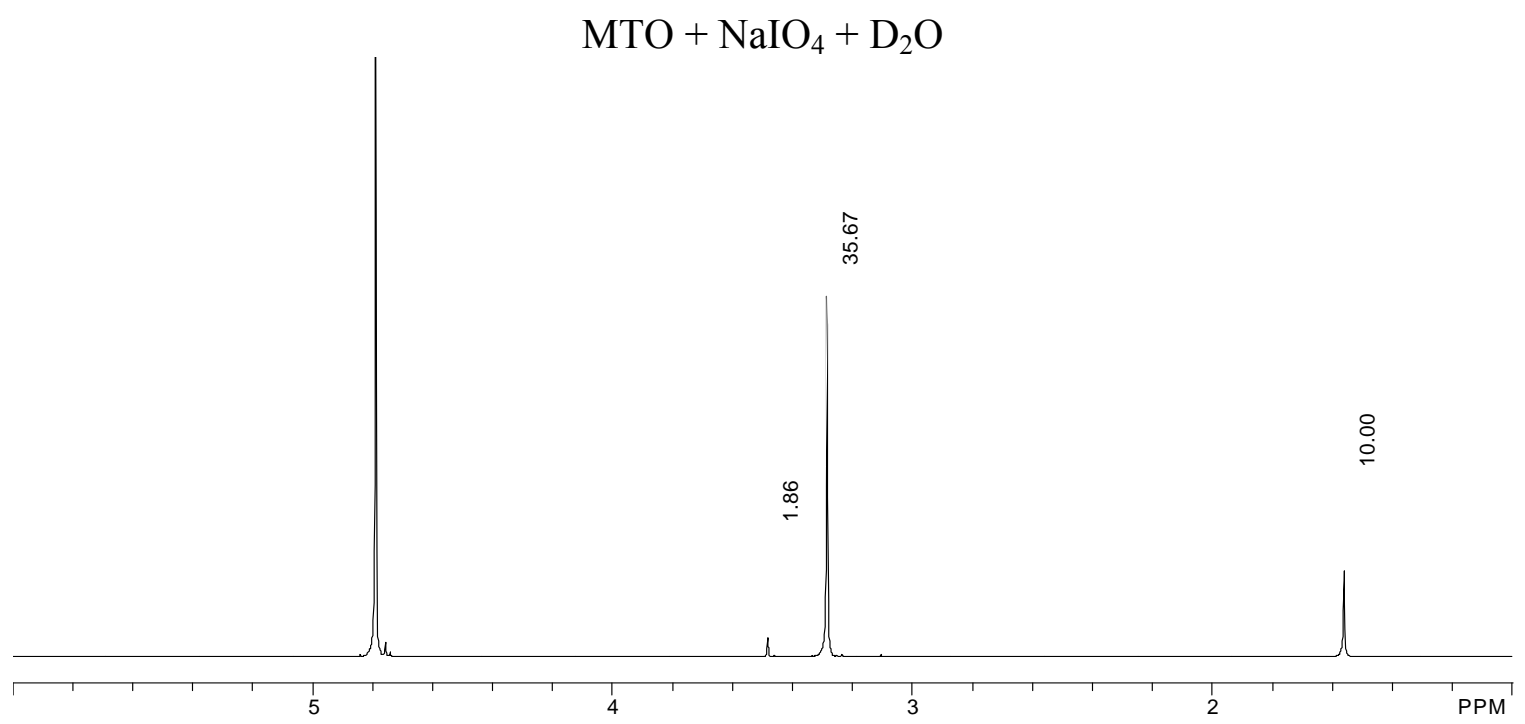

Figure 6: ${ }^{1} \mathrm{H}$ NMR of $\mathrm{MTO}+\mathrm{NaIO}_{4}$ in $\mathrm{D}_{2} \mathrm{O}$ with cyclohexane internal standard: $\delta 1.56$ (cyclohexane); $\delta$ 3.28 (s, $3 \mathrm{H}$, methanol); $\delta 4.79\left(\mathrm{H}_{2} \mathrm{O}\right.$ residual peak).

Theoretical Considerations: All theoretical calculations were performed with the $B 3 \mathrm{LYP}^{2,3}$ density functional, in combination with the Jaguar $6.0^{4}$ computational package. Rhenium and osmium were described with the effective core potential of Hay and Wadt, iodine with the effective core potentials of Ermler and colleagues ${ }^{6}$ while all other atoms used the $6-31 \mathrm{G}^{* * 7}$ all electron basis set. The effects of diffuse functions (namely 6$31 \mathrm{G}^{* *++}$ ) were included with single point calculations. Solvation effects, in water, (computed via single point corrections) were modeled implicitly with the $\mathrm{PCM}^{8,9}$ model $\left(\varepsilon=80.37, r_{\text {solv }}=1.4\right)$.

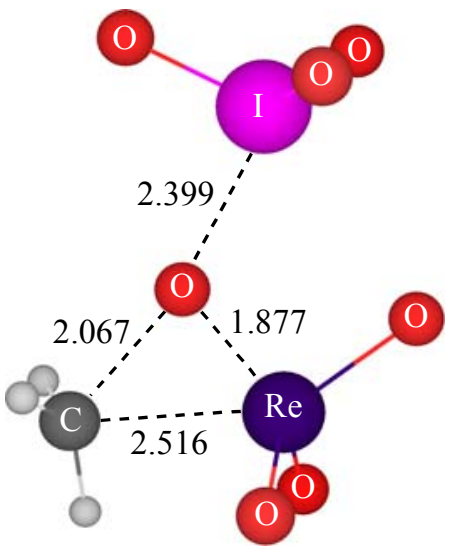

Figure 7: . B3LYP/LACVP/6-311G** BV-type Transition State for MTO + $\mathrm{IO}_{4}^{-}$.

As shown in Figure 7, the BV-type transition state involves concerted methyl migration and $\mathrm{IO}_{3}^{-}$loss as observed by stretching of the $\mathrm{C}$-Re bond from $2.168 \AA$ to $2.516 \AA$ and the I-O bond from $1.803 \AA$ to $2.399 \AA$ 


\section{Cartesian coordinates, enthalpies of key reactants, intermediates and products:}

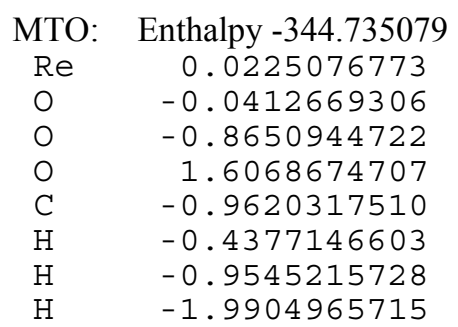

\section{OOH- reaction}

HOOH: Enthalpy -151.524452

$\begin{array}{rr}\mathrm{H} & -1.0954082085 \\ \mathrm{O} & -0.6955460318 \\ \mathrm{O} & 0.6951350748 \\ \mathrm{H} & 1.0951998361\end{array}$

OOH-: Enthalpy -151.094175

$\begin{array}{lr}\mathrm{O} & -0.7310552170 \\ \mathrm{O} & 0.7306760620 \\ \mathrm{H} & 1.0946951166\end{array}$

OH-: Enthalpy -75.971064

$$
\begin{array}{ll}
\mathrm{O} & 0.7302746777 \\
\mathrm{H} & 1.1010653770
\end{array}
$$

$$
\begin{array}{r}
-0.2280123124 \\
0.5330398984
\end{array}
$$

0.9841137885

$-0.7188448601$

1.6111155616

1.6119648144

1.6802485195

1.3083854987

2.7734008924

1.3097371021

Complex: Enthalpy -495.832953

$\begin{array}{lrr}\mathrm{Re} & 0.0298271438 & -0.0255134190 \\ \mathrm{O} & 0.7791697496 & 0.6990804187 \\ \mathrm{O} & -0.3480621300 & -1.6513396528 \\ \mathrm{O} & 1.1841411740 & -0.0772697496 \\ \mathrm{C} & -0.9084243165 & 1.9526485893 \\ \mathrm{H} & -0.0950584448 & 2.6837576753 \\ \mathrm{H} & -1.4685507152 & 2.0070682718 \\ \mathrm{H} & -1.5740067000 & 2.1296874465 \\ \mathrm{O} & -1.7823399141 & -0.2282879115 \\ \mathrm{O} & -2.2045452262 & -1.6020674937 \\ \mathrm{H} & -1.6651819166 & -2.0383345475\end{array}$

-0.1127101386
0.3804764064
0.3803823417
-0.1123842364

0.3801763264 0.3807340958 $-0.1132043404$

0.3812787519 $-0.1218484279$

0.9853726224 $-0.4016481557$

0.3984820841

2. 2620460995

1. 2227400199

1.2000729724

2.1550157327

0.3744139270

1.8730081936

2.1093129530

1. 4026224542

Baeyer-Villager Transiton State: Enthalpy -495.800542

$\begin{array}{lr}\text { Re } & 0.0925144043 \\ \mathrm{O} & -0.5923406510 \\ \mathrm{O} & 1.6806269868 \\ \mathrm{O} & 0.0984742678 \\ \mathrm{C} & -2.1595823735 \\ \mathrm{H} & -2.6995686603 \\ \mathrm{H} & -2.5725467561 \\ \mathrm{H} & -2.0270673552 \\ \mathrm{O} & -0.5029402316 \\ \mathrm{O} & 0.9672094661 \\ \mathrm{H} & 1.5574174855\end{array}$

Product: Enthalpy -419.958234

$\begin{array}{llr}\mathrm{Re} & 0.0960135290 & -0.0252090513 \\ \mathrm{O} & 0.3207157605 & 0.3447293605 \\ \mathrm{O} & 1.5749838015 & 0.0830802637\end{array}$

$$
\begin{array}{r}
-0.0015241948 \\
1.5326356769 \\
0.4701284185 \\
-0.8890690619 \\
0.0884650738 \\
0.0882243655 \\
-0.6169751156 \\
1.0914193806 \\
-1.1387515027 \\
-2.2162263003 \\
-1.6926802475
\end{array}
$$

$$
\begin{array}{r}
0.0083133782 \\
1.6623250847 \\
-0.8394712242
\end{array}
$$


$\begin{array}{ll}\mathrm{O} & -0.5557180356 \\ \mathrm{C} & -2.4434719506 \\ \mathrm{H} & -3.0467614877 \\ \mathrm{H} & -2.8165460393 \\ \mathrm{H} & -2.4762597794 \\ \mathrm{O} & -1.0845566309\end{array}$
$-1.6010380673$

1. 4583523647

0.5509411150

2. 2307213282

1.8177760364

1. 1937849604
$-0.1149101093$

$-0.3570414263$

$-0.4585325560$

$-1.0323814459$

0.6760502544

$-0.7443093694$ 


\section{PyO reaction}

PyO: Enthalpy -323.379837

$\begin{array}{rr}\mathrm{C} & -1.2453415647 \\ \mathrm{C} & 0.1376857259 \\ \mathrm{C} & 0.8606448469 \\ \mathrm{C} & 0.1377958141 \\ \mathrm{C} & -1.2452333159 \\ \mathrm{H} & -1.8754819987 \\ \mathrm{H} & 0.6412578248 \\ \mathrm{H} & 1.9444614065 \\ \mathrm{H} & 0.6414583301 \\ \mathrm{H} & -1.8752944511 \\ \mathrm{~N} & -1.9494849913 \\ \mathrm{O} & -3.2228763298\end{array}$

Py: Enthalpy -248.209568

$\begin{array}{lr}\mathrm{C} & -1.2692437223 \\ \mathrm{C} & 0.1256196330 \\ \mathrm{C} & 0.8378780829 \\ \mathrm{C} & 0.1257009862 \\ \mathrm{C} & -1.2691601068 \\ \mathrm{H} & -1.8544479484 \\ \mathrm{H} & 0.6355253817 \\ \mathrm{H} & 1.9243557612 \\ \mathrm{H} & 0.6357047258 \\ \mathrm{H} & -1.8542958606 \\ \mathrm{~N} & -1.9675430946\end{array}$

Complex: Enthalpy -668.115827

$\begin{array}{lr}\mathrm{Re} & -0.0283353162 \\ \mathrm{O} & 1.4077973818 \\ \mathrm{O} & -0.7203858684 \\ \mathrm{O} & -0.7834254794 \\ \mathrm{O} & -0.2262522282 \\ \mathrm{C} & 1.9263645389 \\ \mathrm{H} & 2.2238926494 \\ \mathrm{H} & 2.6153896524 \\ \mathrm{H} & 1.8761728020 \\ \mathrm{C} & 0.6231529854 \\ \mathrm{C} & 0.1722926673 \\ \mathrm{C} & -0.0610522622 \\ \mathrm{H} & 1.0879725743 \\ \mathrm{C} & -0.5185736486 \\ \mathrm{H} & 0.3039801788 \\ \mathrm{C} & -0.6412627991 \\ \mathrm{H} & -0.1355977339 \\ \mathrm{H} & -0.9587811770 \\ \mathrm{H} & -1.1793041601 \\ \mathrm{~N} & 0.7395875377\end{array}$

$-0.1912252723$

$-0.2051196657$

0.9894878081

2. 1841618802

2. 1703967678

$-1.0700577673$

$-1.1663931709$

0.9894369751

3. 1453876167

3. 0492868349

0.9896184123

0.9896770390

$-0.1526414456$

$-0.2088805465$

0.9895048952

2. 1879409818

2. 1318083715

$-1.0711281496$

$-1.1673526370$

0.9894656194

3. 1463601195

3. 0503385868

0.9896093263

$-0.0733140427$

$-0.4457883853$

0.2978608126

0.9500135419

$-1.7643207189$

0.5164038542

$-0.1000125853$

0.3921580538

1. 5615871146

$-2.2762308891$

$-0.0791814816$

$-2.8035256214$

$-2.8538759817$

$-0.5730646916$

0.9672102254

$-1.9510336707$

$-3.8811732038$

0.1306185122

$-2.3507619180$

$-0.9297256451$
0.0002757594

0.0002519491

$-0.0004860143$

$-0.0011930686$

$-0.0011578157$

0.0008263226

0.0008209650

$-0.0005091501$

$-0.0017836474$

$-0.0016814267$

$-0.0004258756$

$-0.0003983688$

0.0002607430
0.0002601027
-0.0004872800
-0.0011950523
-0.0011244425
0.0008267178
0.0008236424
-0.0005195675
-0.0017910419
-0.0016694354
-0.0004161403

$-0.0126672563$

1.9559748106

$-1.5312043221$

1. 1442046657

0.2258446822

$-0.5679251586$

$-1.4215845614$

0.2645744965

$-0.8872520501$

3. 1280073772

3.8739393289

4. 2104016616

2. 3421701342

4.9676506634

3. 6396881816

5.1505502790

4.3030774794

5.6650911409

6.0029450169

2. 9773588070 
Baeyer-Villager Transition State: Enthalpy -668.064204

$\begin{array}{lrrr}\mathrm{Re} & -0.0085348126 & 0.0290036580 & 0.0706414122 \\ \mathrm{O} & 0.0152669732 & -0.1380269548 & 2.0014431810 \\ \mathrm{C} & 1.9750188822 & 0.3546044552 & 1.3422552660 \\ \mathrm{~N} & -1.5421420243 & -0.5486446055 & 2.7296410086 \\ \mathrm{O} & 0.7883687521 & -1.1709062413 & -0.8638872680 \\ \mathrm{O} & 0.1659723515 & 1.5967691918 & -0.6076644779 \\ \mathrm{O} & -1.6997419577 & -0.3533622093 & 0.0939073618 \\ \mathrm{H} & 2.3407366463 & -0.5453400860 & 1.8238346055 \\ \mathrm{H} & 1.9467833083 & 1.2239316601 & 1.9894633639 \\ \mathrm{H} & 2.4972231267 & 0.5587852592 & 0.4035031909 \\ \mathrm{C} & -1.7614126666 & -1.8240817633 & 3.0400960969 \\ \mathrm{C} & -2.3245601959 & 0.4412178904 & 3.1539240251 \\ \mathrm{C} & -2.8543185891 & -2.1734085416 & 3.8286732307 \\ \mathrm{H} & -1.0565287764 & -2.5456265183 & 2.6421944871 \\ \mathrm{C} & -3.4351880055 & 0.1629536311 & 3.9457465962 \\ \mathrm{H} & -2.0497801776 & 1.4425878913 & 2.8418922604 \\ \mathrm{C} & -3.7016736746 & -1.1637613004 & 4.2868974195 \\ \mathrm{H} & -3.0307278051 & -3.2152588015 & 4.0718550242 \\ \mathrm{H} & -4.0729902378 & 0.9733119815 & 4.2809354368 \\ \mathrm{H} & -4.5609087832 & -1.4081642203 & 4.9029513384\end{array}$

\begin{tabular}{crrr}
\multicolumn{5}{c}{ mu-peroxo Transition State: Enthalpy -668.040308} \\
$\mathrm{Re}$ & 0.0624229735 & 0.0929855350 & 0.1148554018 \\
$\mathrm{O}$ & 0.0933715171 & -0.8053029649 & 1.6800524689 \\
$\mathrm{O}$ & 1.7356600616 & -0.6198029599 & 1.0061822269 \\
$\mathrm{~N}$ & 3.4699882358 & -0.4453954372 & 0.3691885905 \\
$\mathrm{O}$ & -0.8991264370 & -0.7929565791 & -0.9828237174 \\
$\mathrm{O}$ & -0.5891737295 & 1.6434640809 & 0.4071879039 \\
$\mathrm{C}$ & 1.4478843099 & 0.7658959342 & -1.3833084208 \\
$\mathrm{H}$ & 0.8075840941 & 1.2646379865 & -2.1159161738 \\
$\mathrm{H}$ & 1.9623377373 & -0.0716671894 & -1.8531111502 \\
$\mathrm{H}$ & 2.1671784651 & 1.4753709839 & -0.9766532877 \\
$\mathrm{C}$ & 4.0111083681 & -1.4772025588 & -0.2791437339 \\
$\mathrm{C}$ & 4.2270085764 & 0.4976189804 & 0.9307649587 \\
$\mathrm{C}$ & 5.3923698382 & -1.5870294166 & -0.4256780056 \\
$\mathrm{H}$ & 3.3179013542 & -2.2132616043 & -0.6740860070 \\
$\mathrm{C}$ & 5.6157507994 & 0.4583647350 & 0.8272943445 \\
$\mathrm{H}$ & 3.6986644502 & 1.2792150484 & 1.4674375639 \\
$\mathrm{C}$ & 6.2052414845 & -0.6013006229 & 0.1362000026 \\
$\mathrm{H}$ & 5.8141786307 & -2.4296661764 & -0.9626928985 \\
$\mathrm{H}$ & 6.2145620006 & 1.2383136356 & 1.2849218171 \\
$\mathrm{H}$ & 7.2849767733 & -0.6620723931 & 0.0430527563
\end{tabular}

Product: Enthalpy -419.958234 Structure same as $\mathrm{OOH}$ - product. 


\section{$\mathrm{OsO}_{4}(\mathrm{OH})_{2}$ 2- reaction}

$\mathrm{OsO}_{4}(\mathrm{OH})_{2} 2-:$ Enthalpy -543.844876

$\begin{array}{lrrr}\mathrm{OS} & -.7463601841 & .5996390240 & .0220807569 \\ \mathrm{O} & -.6297925986 & .8612937756 & -1.7545424958 \\ \mathrm{O} & -.5140290157 & .9496556223 & 1.7507418610 \\ \mathrm{O} & 1.4084831676 & .7196888233 & -.0424524674 \\ \mathrm{O} & -2.5184996218 & .7418718424 & .0729898417 \\ \mathrm{O} & -.4173134208 & 2.7257489839 & -.0606387923 \\ \mathrm{H} & -.3961198167 & 2.7749937926 & -1.0284129814 \\ \mathrm{O} & -.5081916368 & -1.1459760199 & .0386679090 \\ \mathrm{H} & 1.4301970630 & 1.6794716188 & .0852666098\end{array}$

$\mathrm{OsO}_{3}(\mathrm{OH})_{2}$ 2-: Enthalpy -468.678315

$\begin{array}{lrrr}\text { Os } & -0.6800897024 & 0.6036357758 & -0.2154875116 \\ \mathrm{O} & -0.6867707813 & 0.9177779333 & -2.0469159141 \\ \mathrm{O} & 1.0045949161 & 0.4465539125 & 0.3278899252 \\ \mathrm{O} & -2.3074547253 & 0.3881589607 & 0.3983453198 \\ \mathrm{O} & -0.7064490213 & 2.6136383155 & -0.2956766425 \\ \mathrm{H} & -0.7235324690 & 2.5817253744 & -1.2869006799 \\ \mathrm{O} & -0.5918666638 & -1.5448786697 & -0.1472690360 \\ \mathrm{H} & 0.3573656361 & -1.5983342796 & 0.0321032505\end{array}$

Baeyer-Villager Transition State: Enthalpy -888.535100

$\begin{array}{lrrr}\mathrm{Re} & -0.0679018512 & -0.0504989697 & 0.0491186282 \\ \mathrm{O} & -1.1661509952 & 0.2845631083 & 1.3572655921 \\ \mathrm{O} & 1.5536482973 & 0.2618675710 & 0.5659091221 \\ \mathrm{O} & -0.2920779592 & -1.7171014441 & -0.3663564932 \\ \mathrm{O} & -0.5029519405 & 0.6791722341 & -1.6405316166 \\ \mathrm{C} & -0.4280215210 & 2.3559017877 & -0.7510603283 \\ \mathrm{H} & 0.3784023031 & 2.5597075128 & -0.0439644813 \\ \mathrm{H} & -1.4298894510 & 2.4671110911 & -0.3536192953 \\ \mathrm{H} & -0.2321923847 & 2.7727258127 & -1.7527673699 \\ \mathrm{O} & 1.5386925389 & 0.0243279766 & -3.1813083495 \\ \mathrm{OS} & 0.0936552365 & 0.7539663525 & -3.8256342204 \\ \mathrm{O} & 0.3096046346 & 0.6245031188 & -5.5951423264 \\ \mathrm{O} & 0.3646530192 & 2.5111691359 & -3.5773314371 \\ \mathrm{O} & -0.8936976645 & -1.0567443452 & -4.1418379973 \\ \mathrm{O} & -1.8683336839 & 1.2808223528 & -3.7973694830 \\ \mathrm{H} & -0.7470991948 & -1.1105735630 & -5.1023517755 \\ \mathrm{H} & -2.2291477106 & 0.3787269243 & -3.8195317207\end{array}$

Product: Enthalpy -419.958234 Structure same as $\mathrm{OOH}$ - product

\section{IO $_{4}$ - Reaction}

$\mathrm{IO}_{4}$ : : Enthalpy -412.288013

$\begin{array}{lrrr}\text { I } & -0.3488049918 & 0.3729442403 & -0.0095806512 \\ \mathrm{O} & 0.2665189166 & 1.2826546404 & -1.4392713358\end{array}$




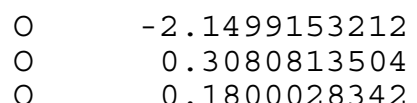

0.3142055189
-1.3057771050
1.2006511465

$\mathrm{IO}_{3}-$ : Enthalpy -337.145366

$\begin{array}{rr}\mathrm{I} & -0.3391880367 \\ \mathrm{O} & 0.2618689636 \\ \mathrm{O} & -2.1582312765 \\ \mathrm{O} & 0.3038257539\end{array}$

$-0.0638167927$

$-0.0372813961$

1.5022063895

0.0178202602

$-1.4573055975$

$-0.0796754760$

$-0.0528848218$

Baeyer-Villager Transition State: Enthalpy -756.996419

$\begin{array}{lr}\text { Re } & 0.0125788072 \\ \mathrm{O} & -0.0534120389 \\ \mathrm{O} & 1.6598328991 \\ \mathrm{O} & -0.2777119564 \\ \mathrm{O} & -0.8569993435 \\ \mathrm{C} & -2.1375939174 \\ \mathrm{H} & -2.5734694769 \\ \mathrm{H} & -2.0523133382 \\ \mathrm{H} & -2.5058240134 \\ \mathrm{I} & 1.5805998896 \\ \mathrm{O} & 0.6240116586 \\ \mathrm{O} & 2.9926767633 \\ \mathrm{O} & 2.1546598663\end{array}$

-0.0317477788
0.0082720311
-0.1326246661
1.6046665450
-1.3820537344
1.2733802799
1.3251801555
2.2126597196
0.4462201087
2.5405607427
3.9792654094
3.1222770178
1.6623581215

mu-peroxo Transition State: Enthalpy -756.964939

$\begin{array}{lr}\mathrm{Re} & -0.0589378784 \\ \mathrm{O} & -0.0604775362 \\ \mathrm{O} & 1.4627757478 \\ \mathrm{I} & 4.0502734276 \\ \mathrm{O} & -0.8314905435 \\ \mathrm{O} & -0.8317733230 \\ \mathrm{C} & 1.4823839491 \\ \mathrm{H} & 0.9351553858 \\ \mathrm{H} & 2.1043224281 \\ \mathrm{H} & 2.1037130463 \\ \mathrm{O} & 4.3726874336 \\ \mathrm{O} & 4.3757652622 \\ \mathrm{O} & 5.2069415444\end{array}$

0.0003845822

$-0.0022140352$

$-0.0001385500$

0.0033312041

$-1.4173712439$

1. 4192945439

0.0021137889

0.0026515109

$-0.8905829802$

0.8951104476

1. 4736518689

$-1.4666957526$

0.0047727063

0.0160164527

1. 7367133655

$-0.4891221793$

$-0.8558098364$

$-0.6062302405$

$-0.0180711596$

$-1.0070006458$

0.5122241572

0.5910786957

$-2.0508271140$

$-2.5811742513$

$-1.0773436265$

$-3.5269952758$

$-0.0153604521$

1.8322308989

1. 2554496647

0.6251010514

$-0.5710615777$

$-0.5675807072$

$-1.4815235915$

$-2.4289955277$

$-1.3863569759$

$-1.3850593024$

$-0.3998671866$

$-0.3993406744$

2. 0230808793

Product: Enthalpy -419.958234

Structure same as $\mathrm{OOH}$ - product

\section{PhIO Reaction}

PhIO: Enthalpy -418.142340

$\begin{array}{ll}\mathrm{C} & -2.5331172743 \\ \mathrm{C} & -1.1508928402 \\ \mathrm{C} & -0.3281927309 \\ \mathrm{C} & -0.8229275879 \\ \mathrm{C} & -2.2074217087 \\ \mathrm{C} & -3.0591784144 \\ \mathrm{H} & -3.1948234494 \\ \mathrm{H} & -0.7415038221\end{array}$

0.6144599249
0.4238865499
1.5423128353
2.8318810894
3.0039132056
1.8991978279
-0.2451974248
-0.5757123678
$-0.0617961204$

0.0188825993

$-0.0580449172$

$-0.2098995559$

$-0.2874632030$

$-0.2150230612$

$-0.0046737500$

0.1385802180 


$\begin{array}{lr}\mathrm{H} & -0.1282214064 \\ \mathrm{H} & -2.6193820817 \\ \mathrm{H} & -4.1347945506 \\ \mathrm{I} & 1.8245299593 \\ \mathrm{O} & 2.2937770171\end{array}$

PhI: Enthalpy -343.019569

$\begin{array}{ll}\mathrm{C} & -2.5292103574 \\ \mathrm{C} & -1.1325406835 \\ \mathrm{C} & -0.4460895929 \\ \mathrm{C} & -1.1324470471 \\ \mathrm{C} & -2.5291042359 \\ \mathrm{C} & -3.2292367927 \\ \mathrm{H} & -3.0659632985 \\ \mathrm{H} & -0.5907046282 \\ \mathrm{H} & -0.5905197053 \\ \mathrm{H} & -3.0658097355 \\ \mathrm{H} & -4.3149104335 \\ \mathrm{I} & 1.6964303864\end{array}$

Complex: Enthalpy -762.887453

$\begin{array}{lr}\mathrm{Re} & -0.1136171847 \\ \mathrm{O} & 0.5058859271 \\ \mathrm{O} & 1.2909028156 \\ \mathrm{O} & -0.6631263436 \\ \mathrm{O} & -0.7402017034 \\ \mathrm{C} & -2.0933796894 \\ \mathrm{H} & -2.3377318370 \\ \mathrm{H} & -2.7932665673 \\ \mathrm{H} & -2.0072398468 \\ \mathrm{I} & 0.7993732932 \\ \mathrm{C} & -0.4829214991 \\ \mathrm{C} & -0.7830409384 \\ \mathrm{C} & -0.9684944832 \\ \mathrm{C} & -1.5620266745 \\ \mathrm{H} & -0.4239349490 \\ \mathrm{C} & -1.7572755032 \\ \mathrm{H} & -0.7454893768 \\ \mathrm{C} & -2.0490663644 \\ \mathrm{H} & -1.7958816839 \\ \mathrm{H} & -2.1414783569 \\ \mathrm{H} & -2.6599089608\end{array}$

3.6684160667 4.0021358626 2. 0377465035 1.4062976055 3. 2194862384

0.4387685805 0.4307501315

1.6459698872

2. 8612315692

2.8533199227

1. 6460730597

$-0.5056010328$

$-0.5088195556$

3. 8007512670

3. 7977169249

1. 6461455571

1. 6458386763

0.4606042883

1. 7311349611

$-0.2260926545$

0.7473893459

$-0.8136438972$

1.4263069046

1. 5405749261

0.7682192360

2. 3872440922

0.0461318866

0.6315260344

$-0.3104461790$

1. 9395046896

0.0709753652

$-1.3325619932$

2. 3051093347

2. 6584664387

1. 3749477150

$-0.6564039256$

3. 3185352679

1.6666933405
$-0.2648751230$

$-0.4067247953$

$-0.2785718398$

0.0495021421

$-0.1044916291$
0.0002127487

0.0006156919

0.0001828524

$-0.0008902187$

$-0.0013423806$

$-0.0007603293$

0.0006343804

0.0015881560

$-0.0012188136$

$-0.0020932946$

$-0.0011219934$

0.0010554152

0.1380386389

1.0961640391

$-0.7856905771$

$-1.7681078828$

1. 0868680222

0.0046520384

1.0635471986

$-0.5031437142$

$-0.4952721889$

$-3.0098384667$

$-4.6334598857$

$-5.6198199055$

$-4.7147336214$

$-6.7155734697$

$-5.5405545969$

$-5.8054544506$

$-3.9336367488$

$-6.8068596198$

$-7.4871695551$

$-5.8740312189$

$-7.6556996266$ 
Baeyer-Villager Transition State: Enthalpy -762.874390

$\begin{array}{lrrr}\mathrm{Re} & 0.1928708004 & -0.3016777024 & -0.1767187369 \\ \mathrm{O} & 1.2324198720 & -0.0915942402 & 1.1717919619 \\ \mathrm{O} & 1.1859973200 & -0.6631682246 & -1.6018958902 \\ \mathrm{O} & -0.3122450370 & 1.2296488538 & -1.2103682050 \\ \mathrm{O} & -0.9330497395 & -1.5781150108 & 0.0382282749 \\ \mathrm{C} & -1.3342839207 & 1.1717809747 & 0.7972955940 \\ \mathrm{H} & -1.3972332617 & 0.5734052067 & 1.7073742463 \\ \mathrm{H} & -2.2490982693 & 1.1870079151 & 0.2184230210 \\ \mathrm{H} & -0.8725836605 & 2.1406404103 & 0.9409032974 \\ \mathrm{I} & 0.7070655384 & 1.2589495901 & -3.1826753009 \\ \mathrm{C} & 2.1258169685 & 0.1038501647 & -4.3062640134 \\ \mathrm{C} & 3.4829110640 & 0.4190794980 & -4.2153636526 \\ \mathrm{C} & 1.6698503945 & -0.9255194031 & -5.1319401307 \\ \mathrm{C} & 4.3955591990 & -0.2954766034 & -4.9929376951 \\ \mathrm{H} & 3.8277364368 & 1.2064198292 & -3.5535152776 \\ \mathrm{C} & 2.5946764567 & -1.6307259071 & -5.9031531558 \\ \mathrm{H} & 0.6154646024 & -1.1758761520 & -5.1777396137 \\ \mathrm{C} & 3.9532498600 & -1.3172197919 & -5.8346208443 \\ \mathrm{H} & 5.4525955962 & -0.0543002916 & -4.9333553385 \\ \mathrm{H} & 2.2498548448 & -2.4290131201 & -6.5534465535 \\ \mathrm{H} & 4.6680395888 & -1.8732857721 & -6.4333489646\end{array}$

Mu-Peroxo Transition State: Enthalpy -762.852895

$\begin{array}{lrrr}\mathrm{Re} & 0.1584963588 & -0.2252561677 & -0.1977264655 \\ \mathrm{O} & -0.1422985097 & -1.0079163833 & 1.3711162699 \\ \mathrm{O} & 1.6689188727 & -0.3106101807 & 1.2637376383 \\ \mathrm{I} & 3.6779057475 & 0.4708184523 & 1.4887971073 \\ \mathrm{O} & 0.3771228085 & -1.4022153266 & -1.4113658015 \\ \mathrm{O} & -1.2543164580 & 0.6843723125 & -0.5148702239 \\ \mathrm{C} & 1.4041575765 & 1.3367334722 & -1.0000491578 \\ \mathrm{H} & 0.8946450388 & 1.6255793919 & -1.9229438528 \\ \mathrm{H} & 2.4079369344 & 0.9865978592 & -1.2351172339 \\ \mathrm{H} & 1.4418331457 & 2.1919274924 & -0.3232835593 \\ \mathrm{C} & 5.0423828702 & 0.1064738130 & -0.1102023200 \\ \mathrm{C} & 5.3651931145 & 1.1490852195 & -0.9835150809 \\ \mathrm{C} & 5.5878953056 & -1.1717226021 & -0.2584899177 \\ \mathrm{C} & 6.2779005748 & 0.9052092917 & -2.0111095601 \\ \mathrm{H} & 4.9229582047 & 2.1323865208 & -0.8641837528 \\ \mathrm{C} & 6.4942530555 & -1.3980249011 & -1.2943774231 \\ \mathrm{H} & 5.3148756749 & -1.9733377042 & 0.4190564664 \\ \mathrm{C} & 6.8407169348 & -0.3628538256 & -2.1653350169 \\ \mathrm{H} & 6.5436280034 & 1.7091924337 & -2.6899905213 \\ \mathrm{H} & 6.9272379163 & -2.3854653869 & -1.4180050302 \\ \mathrm{H} & 7.5484394447 & -0.5459736273 & -2.9675012243\end{array}$

Product: Enthalpy -762.875227

Product Structure Same as OOH- case

\footnotetext{
${ }^{1}$ Pecht, I.; Luz, Z. J. Am. Chem. Soc. 1965, 87, 4068-4072.

${ }^{2}$ Becke, A. D., J. Chem. Phys. 1993, 98, 5648.

${ }^{3}$ Lee, C.; Yang, W.; Parr, R. G., Phys. Rev. B. 1988, 37, 785.

${ }^{4}$ Jaguar 6.0. Schrodinger, LLC: Portland, Oregon, 2005.

${ }^{5}$ Hay, P. J.; Wadt, W. R., J. Chem. Phys. 1985, 82, 299.

${ }^{6}$ LaJohn, L. A.; Christiansen, P. A.; Ross, R. B.; Atashroo, T.; Ermler, W. C., J. Chem. Phys. 1987, 87, 2812.

${ }^{7}$ Harihara, P. C.; Pople, J. A., Theo. Chim. Acta. 1973, 28, 213.

${ }^{8}$ Tannor, D. J.; Marten, B.; Murphy, R.; Friesner, R. A.; Sitkoff, D.; Nicholls, A.; Ringnalda, M.; Goddard, W. A.; Honig, B., J. Am. Chem. Soc. 1994, 116, 11775.
} 
${ }^{9}$ Marten, B.; Kim, K.; Cortis, C.; Friesner, R. A.; Murphy, R.; Ringnalda, M.; Sitkoff, D.; Honig, B., J. Phys. Chem. 1996, 100, 9098. 